\title{
Testing star formation rate indicators using galaxy merger simulations and radiative transfer
}

\author{
Christopher C. Hayward ${ }^{1,2}$, Patrik Jonsson ${ }^{1}$, Kai Noeske ${ }^{1}$, Stijn \\ Wuyts $^{1}$, T.J. Cox ${ }^{3}$, Desika Narayanan ${ }^{1}$, Brent Groves ${ }^{4} \&$ Lars \\ Hernquist $^{1}$ \\ ${ }^{1}$ Harvard-Smithsonian Center for Astrophysics, 60 Garden Street, Cambridge, MA 02138, USA \\ ${ }^{2}$ chayward@cfa.harvard.edu \\ ${ }^{3}$ Carnegie Observatories, 813 Santa Barbara Street, Pasadena, CA 91101, USA \\ ${ }^{4}$ Sterrewacht Leiden, Leiden University, Niels Bohrweg 2, Leiden 2333-CA, The Netherlands
}

\begin{abstract}
We discuss our ongoing project analyzing N-body/smoothed-particle hydrodynamics simulations of isolated and merging galaxies, performed using GADGET-2 (Springel 2005), with the 3-D adaptive grid, polychromatic Monte Carlo radiative transfer code SUNRISE (Jonsson 2006). We apply commonly used UV, optical, and IR star formation rate (SFR) indicators to the integrated spectral energy distributions (SEDs) of the simulated galaxies in order to determine how well the SFR indicators recover the instantaneous SFR in the simulations. The models underlying each SFR indicator must necessarily make assumptions about physical properties of the galaxies, e.g., the star formation history $(\mathrm{SFH})$, whereas all such properties are known in the simulations. This enables us to test and compare SFR indicators in a way that is complementary to observational studies. We present one preliminary result of interest: even after correcting the $\mathrm{H} \alpha$ luminosity for dust using the Calzetti et al. (2000) attenuation law the SFR is significantly underestimated for simulated galaxies with SFR $\gtrsim 10 M_{\odot} \mathrm{yr}^{-1}$.
\end{abstract}

Keywords. stars: formation, galaxies: stellar content, galaxies: fundamental parameters, galaxies: starburst, galaxies: high-redshift, dust, extinction, radiative transfer

\section{Introduction}

To truly understand galaxy formation we must understand star formation, as stars account for a significant fraction of the visible mass of a galaxy, deplete and enrich the gas reservoirs present in galaxies, and are an important source of feedback. The starformation histories (SFH) and instantaneous star-formation rates (SFR) of individual galaxies provide key information about their formation histories, and comparisons of the SFHs and SFRs of different galaxy populations can elucidate the relationships among the populations.

Unfortunately the SFR cannot be observed directly but rather must be inferred from broad-band photometry or spectra. In principle, various parts of the electromagnetic spectrum can be used to measure the SFR (see Kennicutt 1998 for a review). However, some indicators work better than others, and some should only be applied to certain galaxy populations. Since the SFR cannot be measured directly, the relationship between an indicator and the SFR must be calibrated using theoretical models. Such calibrations are difficult because they rely on the IMF, details of stellar population synthesis models, and the star-formation history assumed. Uncertainties in each of these components lead to uncertainties in the SFR indicator calibration. When applying an indicator to galaxies, contamination from older stellar populations and AGN can cause 
inaccurate SFR estimates. Furthermore, one must correct UV and optical SFR indicators for dust attenuation or the SFR will be significantly underestimated.

Current topics for which accurate understanding of SFR indicators and dust corrections is necessary include the cosmic mass-assembly history (e.g., Bouwens et al. 2009; Reddy \& Steidel 2009), the SFR-stellar mass relation (e.g., Noeske et al. 2007a,b; Daddi et al. 2007), the relationship among the so-called red sequence, blue cloud, and green valley (e.g., Brammer et al. 2009), and the nature of sub-millimeter galaxies (e.g., Narayanan et al. 2009a,b; Davé et al. 2009). We aim to characterize the systematic and random uncertainties inherent in standard SFR indicators and dust corrections in order to understand when apparent discrepancies between the SFR in models and observations are true discrepancies rather than just the result of inaccurate SFR estimates. We also hope to derive more robust SFR indicators and dust corrections, paying particular attention to high- $z$ galaxies (as simulated in, e.g., Narayanan et al. 2009b,c).

\section{Methodology}

We utilize a large suite of GADGET-2 (Springel 2005) $N$-body/SPH simulations of isolated disk and merging galaxies including the two-phase sub-resolution ISM model of Springel \& Hernquist (2003) and black hole accretion and feedback (Springel et al. 2005). See, e.g., Cox et al. (2006) for details.

We perform dust radiative transfer on the GADGET simulations in postprocessing using the 3-D adaptive grid polychromatic Monte Carlo radiative transfer code SUNRISE (Jonsson 2006; Jonsson et al. 2009). Star particles older than 10 Myr are assigned singleage stellar population SEDs from the 2006 version of STARBURST99 (Leitherer et al. 1999; Smith et al. 2002; Vázquez \& Leitherer 2005) using a Kroupa (2001) IMF. The SEDs assigned to star particles younger than $10 \mathrm{Myr}$ account for the effects of gas and dust in the HII and photodissociation regions (PDRs) around the clusters by using the SED templates of Groves et al. (2008). Black hole particles are assigned luminosity-dependent templates from Hopkins et al. (2007).

SUNRISE performs full radiative transfer for $\sim 1000$ wavelengths in the range $\sim 1000 \AA$ $-1 \mathrm{~mm}$. We calculate spatially-resolved SEDs for seven viewing angles and for temporal snapshots spaced $10 \mathrm{Myr} / h$ apart spanning the $\sim 2$ Gyr duration of the simulations. For the simulations presented here we use the dust model of Weingartner \& Draine (2001) with the updates of Draine \& Li (2007) assuming a dust-to-metal ratio of 0.4 (Dwek 1998). Dust self-absorption and re-emission are calculated through an iterative process (Juvela 2005); this is key to accurately treat the high dust densities found in ULIRGs (Younger et al. 2009). The polychromatic nature of SUNRISE enables study of, e.g., H $\alpha$ emission from our simulated galaxies, which is key to the present work.

\section{Results}

Here we show an example of the analysis to be presented in Hayward et al. (2010, in preparation). In Figure 1 we plot the $\mathrm{H} \alpha$ luminosity measured from our simulated integrated SEDs versus the instantaneous SFR for a set of major merger simulations. The solid line is the Kennicutt (1998) calibration corrected for the Kroupa (2001) IMF used in our simulations. The dashed line is the best fit to our data, where we have assumed $\log L(\mathrm{H} \alpha)=\log \mathrm{SFR}+C$. As expected, the $\mathrm{H} \alpha$ luminosity is roughly linearly proportional to the SFR. However, without correcting for dust the $\mathrm{H} \alpha$ luminosity is significantly $(\sim 4.5 \mathrm{x})$ less than expected from the standard calibration, and there is a large dispersion at given SFR owing to different viewing angles. 


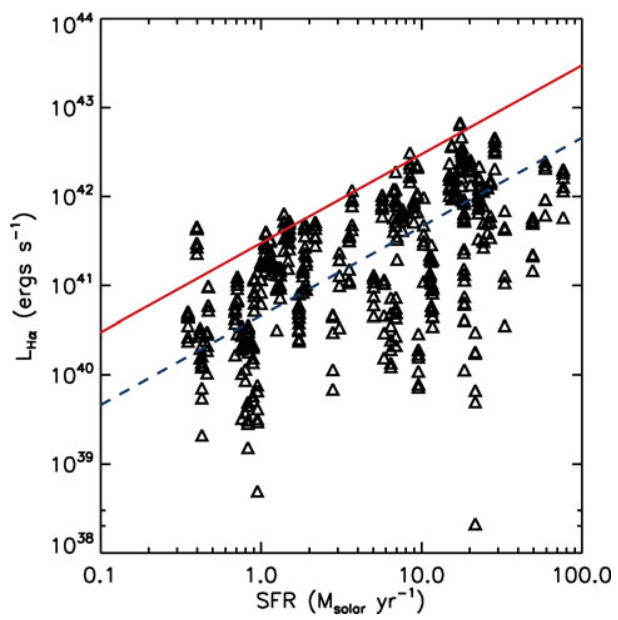

Figure 1. $\mathrm{H} \alpha$ luminosity $L(\mathrm{H} \alpha)$ uncorrected for dust in ergs $\mathrm{s}^{-1}$ versus instantaneous SFR in $M_{\odot} \mathrm{yr}^{-1}$ for a set of major merger simulations. The solid line is the calibration of Kennicutt (1998) converted to the Kroupa (2001) IMF we employ. The dashed line is the best fit to our simulation data. The observed (attenuated) $\mathrm{H} \alpha$ is significantly underluminous and shows as much as 2-3 orders of magnitude scatter.

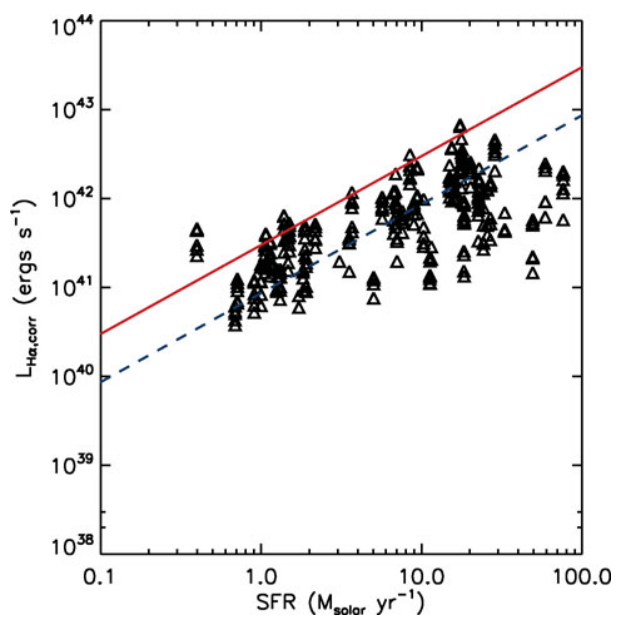

Figure 2. $L(\mathrm{H} \alpha)$ corrected for dust using the Calzetti et al. (2000) attenuation law versus instantaneous SFR. The solid red line is the Kennicutt (1998) calibration corrected for the difference in IMF, and the dashed blue line is the best fit to our simulated data. While correcting for dust attenuation reduces the scatter and systematic error in the relation, the $\mathrm{H} \alpha$ luminosity is still less than expected from the standard calibration. This problem is more severe at SFR $\gtrsim 10 M_{\odot} \mathrm{yr}^{-1}$.

In Figure 2 we plot the dust-corrected $\mathrm{H} \alpha$ luminosity versus instantaneous SFR. We have corrected for dust by measuring the color excess via the $\mathrm{H} \beta / \mathrm{H} \alpha$ ratio and using the Calzetti et al. (2000) attenuation law. Correcting for dust in this manner reduces both the scatter and systematic error in the correlation, but $\mathrm{H} \alpha$ is still underluminous, especially for $\mathrm{SFR} \gtrsim 10 M_{\odot} \mathrm{yr}^{-1}$. The best fit is $\sim 3 \mathrm{x}$ less than the Kennicutt (1998) calibration converted to the Kroupa (2001) IMF. Some of the discrepancy owes to the different stellar population synthesis models we use, but this is $\lesssim 30 \%$. The rest owes to under-correcting for dust attenuation. This under-correction is not necessarily surprising (and has been 
observed by others, e.g., Jonsson et al. 2006), as the law was empirically derived from a sample of starburst galaxies with relatively modest SFR compared to those encountered in our simulations. At high SFR the central, most rapidly star-forming regions are highly obscured, so they contribute less to the resulting Balmer line flux. Thus the $\mathrm{H} \beta / \mathrm{H} \alpha$ ratio may not adequately characterize the attenuation suffered by the most heavily obscured star-forming regions. Since the opacity at $\mathrm{Pa} \alpha$ is less than at $\mathrm{H} \beta$, use of $\mathrm{Pa} \alpha / \mathrm{H} \alpha$ may better recover the attenuation and result in a superior dust correction. As Pa $\alpha$ data for large samples low- $z$ galaxies are becoming increasingly available, and it is possible to detect $\mathrm{Pa} \alpha$ from lensed high- $z$ galaxies (Papovich et al. 2009), this is a promising solution and will be investigated as part of our complete work.

\section{References}

Bouwens, R. J., et al. 2009, arXiv:0909.4074

Brammer, G. B., et al. 2009, arXiv:0910.2227

Calzetti, D., Armus, L., Bohlin, R. C., Kinney, A. L., Koornneef, J., \& Storchi-Bergmann, T. 2000, ApJ, 533, 682

Cox, T. J., Dutta, S. N., Matteo, T. D., Hernquist, L., Hopkins, P. F., Robertson, B., \& Springel, V. 2006, ApJ, 650, 791

Daddi, E., et al. 2007, ApJ, 670, 156

Davé, R., Finlator, K., Oppenheimer, B. D., Fardal, M., Katz, N., Kereš, D., \& Weinberg, D. H. 2009, arXiv:0909.4078

Draine, B. T., \& Li, A. 2007, ApJ, 657, 810

Dwek, E. 1998, ApJ, 501, 643

Groves, B., Dopita, M. A., Sutherland, R. S., Kewley, L. J., Fischera, J., Leitherer, C., Brandl, B., \& van Breugel, W. 2008, ApJS, 176, 438

Hopkins, P. F., Richards, G. T., \& Hernquist, L. 2007, ApJ, 654, 731

Jonsson, P. 2006, MNRAS, 372, 2

Jonsson, P., Cox, T. J., Primack, J. R., \& Somerville, R. S. 2006, ApJ, 637, 255

Jonsson, P., Groves, B., \& Cox, T. J. 2009, arXiv:0906.2156

Juvela, M. 2005, $A \& A$, 440, 531

Kennicutt, R. C. 1998, ARAA, 36, 189

Kroupa, P. 2001, MNRAS, 322, 231

Leitherer, C., et al. 1999, ApJS, 123, 3

Narayanan, D., Cox, T. J., Hayward, C., Younger, J. D., \& Hernquist, L. 2009a, arXiv:0905.2184

Narayanan, D., Hayward, C. C., Cox, T. J., Hernquist, L., Jonsson, P., Younger, J. D., \& Groves, B. 2009b, arXiv:0904.0004

Narayanan, D., et al. 2009c, arXiv:0910.2234

Noeske, K. G., et al. 2007a, ApJ, 660, L47

-. 2007b, ApJ, 660, L43

Papovich, C., Rudnick, G., Rigby, J. R., Willmer, C. N. A., Smith, J.-D. T., Finkelstein, S. L., Egami, E., \& Rieke, M. 2009, ApJ, 704, 1506

Reddy, N. A. \& Steidel, C. C. 2009, ApJ, 692, 778

Smith, L. J., Norris, R. P. F., \& Crowther, P. A. 2002, MNRAS, 337, 1309

Springel, V. 2005, MNRAS, 364, 1105

Springel, V. \& Hernquist, L. 2003, MNRAS, 339, 289

Springel, V., Matteo, T. D., \& Hernquist, L. 2005, MNRAS, 361, 776

Vázquez, G. A. \& Leitherer, C. 2005, ApJ, 621, 695

Weingartner, J. C. \& Draine, B. T. 2001, ApJ, 548, 296

Younger, J. D., Hayward, C. C., Narayanan, D., Cox, T. J., Hernquist, L., \& Jonsson, P. 2009, MNRAS, 396, L66 